\section{Skin biomarkers for neurodegenerative disease: a future perspective}

\author{
“...skin biopsies may represent an alternative to support the diagnosis of \\ Parkinson's disease and Alzheimer's disease, the two most common \\ neurodegenerative diseases. The finding of a-synuclein and tau protein \\ by immunohistochemistry using commercially available antibodies \\ could support the diagnosis.”
}

Juan Pablo Castanedo-Cazares*,1 \& Idelfonso Rodriguez-Leyva²

\section{Disease Management}

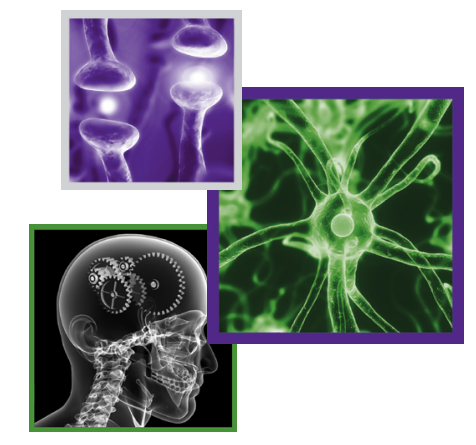

\section{A biomarker represents a chateristic that}

A biomarker represents a characteristic that can be objectively measured and evaluated as an indicator of normal or pathogenic processes, or of pharmacologic responses to a therapeutic intervention [1]. Biomarkers essentially pretend to confirm a diagnosis, being useful for epidemiological screening, predictive testing, monitoring of disease progression after diagnosis, drug development and its response to treatment [1].

A major hallmark of neurodegenerative diseases is the abnormal deposition of aggregates of misfolded proteins; such diseases are called proteinopathies [2]. This phenomenon initiates a series of intracellular perturbations that lead to cell dysfunction and eventually cell death, with a corresponding progressive impairment in neuronal function $[2,3]$. These illnesses are initially difficult to diagnose, as distinctive signs and symptoms appear progressively until the advanced stages. They are usually accompanied clinically by dementia, parkinsonism and/or motor dysfunction [3-5].

In general, manifestations of these diseases are secondary to the aggregation of defectively folded proteins in different brain regions. These proteins are able to evade degradation mechanisms and initiate a series of neurotoxic effects, including synaptic dysfunction and disruption of cellular organelles and the cytoskeleton, enabling an inflammatory response and ultimately leading to cell death [6]. In terms of the positioning of these protein aggregates, recent evidence supports the notion that they are spread among the cells of the nervous system in a similar way to prions [7].

The identification of an accessible tissue biomarker prior to symptom development will allow the identification of individuals in the very earliest stages of disease, and could provide a means of assessing whether particular therapies could modify the continuing pathological process. In the search for potential histological markers, the acquisition of any nervous tissue is still currently a complex and hazardous intervention in humans. Thus, other tissues have been evaluated as possible surrogates, including those of the salivary glands, olfactory epithelium, heart and digestive tract $[8,9]$.

As much in terms of the diagnosis as of the treatment of a proteinopathy, the principal advances that have been made are in Parkinson's disease (PD), where the presence

\section{KEYWORDS}

- Alzheimer disease $\bullet$ biomarkers

- neurodegenerative diseases

- Parkinson disease • skin

'Hospital Central Dr. Ignacio Morones Prieto, Dermatology Department, Universidad Autónoma de San Luis Potosí, San Luis Potosí, SLP, Mexico

${ }^{2}$ Hospital Central Dr. Ignacio Morones Prieto, Neurology Department, Universidad Autónoma de San Luis Potosí, San Luis Potosí, SLP, Mexico

*Author for correspondence: Tel.: +52 4448342795; Fax: +52 4448342795; castanju@yahoo.com 


\section{"The identification of an accessible tissue biomarker prior to symptom development will allow the identification of individuals in the very earliest stages of disease..."}

of $\alpha$-synuclein forms insoluble neurofibrillary tangles distributed throughout the body, forming Lewy neurites. In the neurons they form conglomerate eosinophilic inclusions surrounded by a clear halo known as Lewy bodies $[4,8]$. A relationship between the brain and the skin was proposed by Makrantonaki et al. and finally reported by our working team, after examining the expression of $\alpha$-synuclein in the epidermis and skin's appendages: expression was moderate in PD, mild in atypical parkinsonism and null in healthy control subjects $[10,11]$.

Neural and epidermal tissues share a common embryological origin, as ectodermal derivatives. So, it is anticipated than both may accumulate similar abnormal misfolded proteins related to degenerative processes [11]. Studies have shown that genes expressed in signaling pathways operating in age-associated neurodegenerative disease, such as Huntington's disease, dentatorubralpallidoluysian atrophy, amyotrophic lateral sclerosis and PD, are expressed in hormonally aged human sebocytes $[10,12]$. Therefore, cutaneous tissue is a promising source in the search for biological markers of neurodegenerative conditions. This is not due exclusively to its ontological origin, but also to its profuse innervations and its capacity to respond to, produce and release diverse neuropeptides [12,13].

Cutaneous tissue also expresses genes involved in neurological diseases, such as APP, tau PSEN1 and PARK2, among others [10]. Accordingly, several diseases of the nervous system have dermal manifestations, as is the case with PD, where there is an increased risk of melanoma and patients frequently present seborrhea and hyperhidrosis alongside the classical motor manifestations of the disease [14].

Despite the initial failure to observe Lewy pathology using routine biopsies from cadaveric skin [15], we were able to detect it in skin samples of patients with PD compared with healthy individuals [11]. The discrepancy between the results of the autopsy-based study and the in vivo study may be explained by differences in the sites of the obtained samples, the size and number of examined sections, the type of antibodies used for $\alpha$-synuclein (phosphorylated/non-phosphorylated) and the histological processing (fresh frozen or paraffin-embedded tissue). Moreover, $\alpha$-synuclein aggregates have also been recently demonstrated in peripheral nerve terminals of the epidermis and skin appendages of PD patients [16]. The presence of protein aggregates in skin cells as a potential biomarker of disease has also recently been demonstrated for Alzheimer's disease. The phosphorylated tau protein was detected by immunohistochemistry in $71 \%$ of the studied patients [17].

Biomarkers of neurodegenerative diseases have been developed in recent years; however, they are mainly based on advanced molecular neuroimaging. A major drawback is that worldwide, most health systems do not have the capacity to offer these tests in clinical practice. Therefore, skin biopsies may represent an alternative to support the diagnosis of PD and Alzheimer's disease, the two most common neurodegenerative diseases. The finding of $\alpha$-synuclein and tau protein by immunohistochemistry using commercially available antibodies could support the diagnosis. This can be easily done in regularly equipped pathology laboratories worldwide. It is possible that in the near future we will be able to characterize in the skin signs of other neurodegenerative diseases such as dementia with Lewy bodies, fronto-temporal lobar degeneration, amyotrophic lateral sclerosis, Huntington's disease and the prion diseases. All of these disorders are characterized as genetically complex proteinopathies, and reflect a Mendelian inheritance modified by epigenetic factors.

\section{Financial \& competing interests disclosure}

The authors have no relevant affiliations or financial involvement with any organization or entity with a financial interest in or financial conflict with the subject matter or materials discussed in the manuscript. This includes employment, consultancies, honoraria, stock ownership or options, expert testimony, grants or patents received or pending, or royalties.

No writing assistance was utilized in the production of this manuscript.

\section{References}

Biomarkers Definitions Working Group.

Biomarkers and surrogate end points:

preferred definitions and conceptual

framework. Clin. Pharmacol. Ther. 69(3), 89-95 (2001).
2 Wakabayashi K, Tanji K, Mori F. Pathology of basal ganglia in neurodegenerative diseases. Brain Nerve 61(4), 429-439 (2009).

3 Huang Y, Mucke L. Alzheimer mechanisms and therapeutic strategies. Cell 148(6), 1204-1222 (2012).
4 Trojanowski JQ, Lee VM. Aggregation of neurofilament and alpha-synuclein proteins in Lewy bodies: implications for the pathogenesis of Parkinson disease and Lewy body dementia. Arch. Neurol. 55(2), 151-152 (1988). 
5 Mollenhauer B, Rochester L, Chen-Plotkin A, Brooks D. What can biomarkers tell us about cognition in Parkinson's disease? Mov. Disord. 29(5), 622-633 (2014).

6 Lee SJ, Lim HS, Masliah E, Lee HJ. Protein aggregate spreading in neurodegenerative diseases: problems and perspectives. Neurosci. Res. 70(4), 339-348 (2011).

7 Goedert M, Clavaguera F, Tolnay M. The propagation of prion-like protein inclusions in neurodegenerative diseases. Trends Neurosci. 33(7), 317-325 (2010).

8 Beach TG, Adler CH, Sue LI et al. Multiorgan distribution of phosphorylated alpha-synuclein histopathology in subjects with Lewy body disorders. Acta Neuropathol. 119(6), 689-702 (2010).

9 Lebouvier T, Tasselli M, Paillusson S, Pouclet $\mathrm{H}$, Neunlist M, Derkinderen P. Biopsable neural tissues: Toward new biomarkers for Parkinson's disease? Front. Psychiatry 1, 128 (2010).

10

Makrantonaki E, Brink TC, Zampeli V et al. Identification of biomarkers of human skin ageing in both genders. Wnt signalling - a label of skin ageing? PLoS ONE 7(11), e50393 (2012).

11 Rodr guez-Leyva I, Calderón-Garcidueñas AL, Jiménez-Capdeville ME et al. $\alpha$-synuclein inclusions in the skin of Parkinson's disease and parkinsonism. Ann. Clin. Trans. Neurol. 1(7), 213-215 (2014).

12 Zouboulis CC, Baron JM, Böhm M et al. Frontiers in sebaceous gland biology and pathology. Exp. Dermatol. 17(6), 542-551 (2008).

13 Clos AL, Kayed R, Lasagna-Reeves CA. Association of skin with the pathogenesis and treatment of neurodegenerative amyloidosis. Font. Neurol. 3, 5 (2012).

14 Antonini A, Barone P, Marconi R et al. The progression of non-motor symptoms in Parkinson's disease and their contribution to motor disability and quality of life. J. Neurol. 259(12), 2621-2631 (2012).

15 Miki Y, Tomiyama M, Ueno T et al. Clinical availability of skin biopsy in the diagnosis of Parkinson's disease. Neurosci. Lett. 469(3), 357-359 (2010).

16 Wang N, Gibbons CH, Lafo J, Freeman BSR $\alpha$-Synuclein in cutaneous autonomic nerves. Neurology 81(18), 1604-1610 (2013).

17 Rodriguez-Leyva I, Chi-Ahumada E, Calderon-Garcidueñas L et al. Presence of phosphorylated tau protein in the skin of Alzheimer's disease patients. J. Mol. Biomark. Diagn. s6, 005 (2015). 